\title{
The Dynamics of Component Separation in a Binary Mixture of Bose-Einstein Condensates
}

\author{
D. S. Hall, M. R. Matthews, J. R. Ensher, C. E. Wieman, and E. A. Cornellt \\ JILA, National Institute of Standards and Technology and Department of Physics, University of Colorado, \\ Boulder, Colorado 80309-0440
}

(April 2, 1998)

\begin{abstract}
We present studies of the time-evolution of a twocomponent system of Bose-Einstein condensates (BEC) in the $\left|F=1, m_{f}=-1\right\rangle$ and $|2,1\rangle$ spin states of ${ }^{87} \mathrm{Rb}$. The two condensates are created with a well-defined relative phase and complete spatial overlap. In subsequent evolution they undergo complex relative motions that tend to preserve the total density profile. The motions quickly damp out, leaving the condensates in a steady state with a nonnegligible (and adjustable) overlap region.
\end{abstract}

Since its realization in dilute atomic gases [1] 3], BoseEinstein condensation (BEC) has afforded an intriguing glimpse into the macroscopic quantum world. Attention has recently broadened to include exploration of systems of two or more condensates, as realized in a magnetic trap in rubidium [4] and subsequently in an optical trap in sodium [5]. Theoretical treatment of such systems began in the context of superfluid helium mixtures [6] and spinpolarized hydrogen [7], and has now been extended to $\mathrm{BEC}$ in the alkalis [8]10].

The first experiments involving the interactions between multiple-species BEC were performed with atoms evaporatively cooled in the $\left|F=2, m_{f}=2\right\rangle$ and $|1,-1\rangle$ spin states of ${ }^{87} \mathrm{Rb}$ 䏽. These experiments demonstrated the possibility of producing long-lived multiple condensate systems, and that the condensate wavefunction is dramatically affected by the presence of interspecies interactions. In this Letter, we report results from initial studies of simultaneously trapped BECs in the $|2,1\rangle$ and $|1,-1\rangle$ states of ${ }^{87} \mathrm{Rb}$ (denoted hereafter as $|2\rangle$ and $|1\rangle$, respectively). The two states are completely distinguishable since the hyperfine splitting is much larger than any other relevant energy scale in the system. We produce arbitrary superpositions of $|1\rangle$ and $|2\rangle$ that begin with a well-defined relative phase, spatial extent, and "sag" the position at which the magnetic trapping forces balance gravity for each state. The fine experimental control of this double condensate system permits us to study its subsequent time-evolution under a variety of interesting conditions, most notably those in which there remains substantial overlap between the two states.

The apparatus and general procedure we use to attain $\mathrm{BEC}$ in $\mathrm{Rb}$ are identical to those of our previous work [1] and will be reviewed here but briefly. We use a

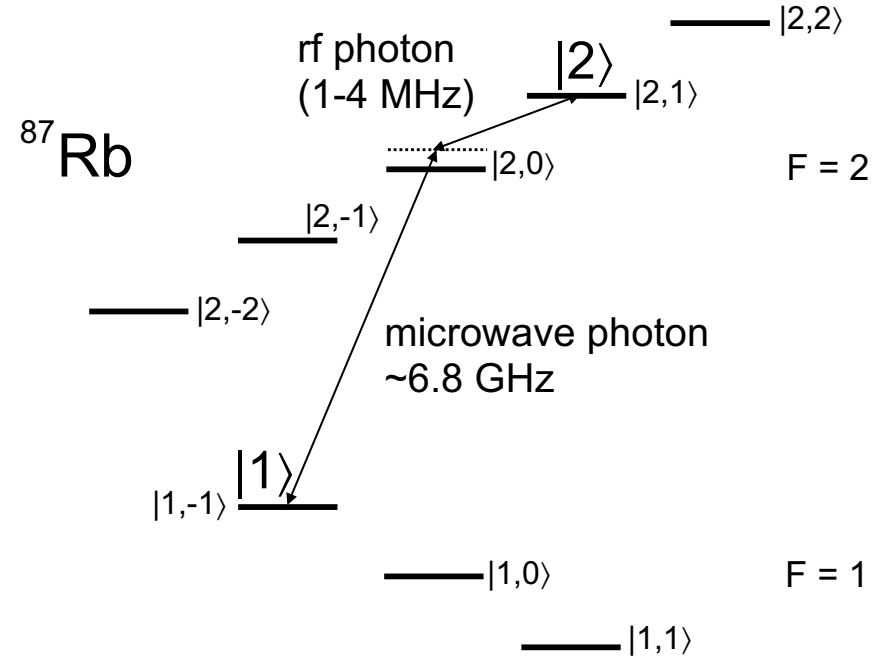

FIG. 1. A schematic of the ground-state hyperfine levels $(F=1,2)$ of ${ }^{87} \mathrm{Rb}$, shown with a splitting due to an applied magnetic field. The two-photon transition is driven between the $|1,-1\rangle(|1\rangle)$ and $|2,1\rangle(|2\rangle)$ states.

double magneto-optical trap system to load roughly $10^{9}$ $|1\rangle$ atoms into a time-averaged, orbiting potential (TOP) magnetic trap [12]. The atoms are magnetically compressed and evaporatively cooled [1] for $30 \mathrm{~s}$ until they form a condensate of approximately $5 \times 10^{5}$ atoms with no noticeable non-condensate fraction (we estimate that $>75 \%$ of the entire gas is in the condensate). After completion of the evaporation cycle, the magnetic trap is ramped adiabatically to various bias fields and spring constants for the subsequent experiments.

The double condensate system is prepared from the single $|1\rangle$ condensate by driving a two-photon transition 111 consisting of a microwave photon near $6.8 \mathrm{GHz}$ and a radiofrequency (rf) photon of $1-4 \mathrm{MHz}$, depending on the Zeeman splitting (Fig. 11). As in [11], we are able to transfer quickly any desired fraction of the atoms to the $|2\rangle$ state by selecting the length and amplitude of the two-photon pulse. The two condensates are created with identical density distributions, after which they evolve and redistribute themselves for some time $T$. We then turn off the magnetic trap and allow the atoms to expand for $22 \mathrm{~ms}$ for imaging.

We selectively image the densities of either of the two states $\left(n_{1}\right.$ and $\left.n_{2}\right)$ or the combined density distribution 
$\left(n_{T}\right)$ by changing the sequence of laser beams applied to the condensates for probing [11]. Since the expansion and imaging are destructive processes, each image is taken with a different condensate; the excellent reproducibility of the condensates permits us to study the time-evolution of the system by changing the time $T$. We subsequently use a least-squares algorithm to reconstruct the relative positions of the condensates from the images of $n_{1}, n_{2}$, and $n_{T}$ at each time $T$ - a task made necessary by shotto-shot jitter in the image positions on the CCD-array detector.

The evolution of the double condensate system, including the release from the trap and subsequent expansion [1, 13], is governed by a pair of coupled GrossPitaevskii equations for condensate amplitudes $\Phi_{i}$ :

$$
i \hbar \frac{\partial \Phi_{i}}{\partial t}=\left(-\frac{\hbar^{2} \nabla^{2}}{2 m}+V_{i}+U_{i}+U_{i j}\right) \Phi_{i}
$$

where $i, j=1,2(i \neq j), V_{i}$ is the magnetic trapping potential for state $i$, the mean-field potentials are $U_{i}=$ $4 \pi \hbar^{2} a_{i}\left|\Phi_{i}\right|^{2} / m$ and $U_{i j}=4 \pi \hbar^{2} a_{i j}\left|\Phi_{j}\right|^{2} / m, m$ is the mass of the $\mathrm{Rb}$ atom, and the intraspecies and interspecies scattering lengths are $a_{i}$ and $a_{i j}$. In the Thomas-Fermi limit, the condensate density distributions are dominated by the potential energy terms of Eq. (11). Consequently, the expanded density distributions retain their spatial information and emerge with their gross features (such as the relative position of the condensates) intact.

The similarity in scattering lengths $a_{1}, a_{2}$, and $a_{12}$ implies that the total density $n_{T}$ will not change significantly from its initial configuration even though the two components may redistribute themselves dramatically during the evolution time $T$. In ${ }^{87} \mathrm{Rb}$, the scattering lengths are known at the $1 \%$ level to be in the proportion $a_{1}: a_{12}: a_{2}:: 1.03: 1: 0.97$, with the average of the three being 55(3) $\AA$ [14, 11]. The near-preservation of the total density $n_{T}$ can be approached theoretically by deriving from Eq. (11) the hydrodynamic equations of motion [15] for $n_{T}$ and evaluating them in the limit that the fractional differences between the scattering lengths are small. The pressures that tend to redistribute $n_{T}$ must also be small. A similar argument pertains if the minima of the trapping potentials $V_{1}$ and $V_{2}$ are displaced from one another (see below) by a distance that is small compared to the size of the total condensate; once again, the effects on the equilibrium distribution of the individual components may be profound but the total density should remain largely unperturbed [16].

The rotating magnetic field of the TOP trap [12] gives rise to a subtle behavior that permits us to displace the minima of the trapping potentials $V_{1}$ and $V_{2}$ with respect to one another [17, 18]. In the rotating frame, the two states see two different magnetic fields as a function of the bias field rotation frequency and sense of rotation (as well as the strengths of the bias and quadrupole fields).

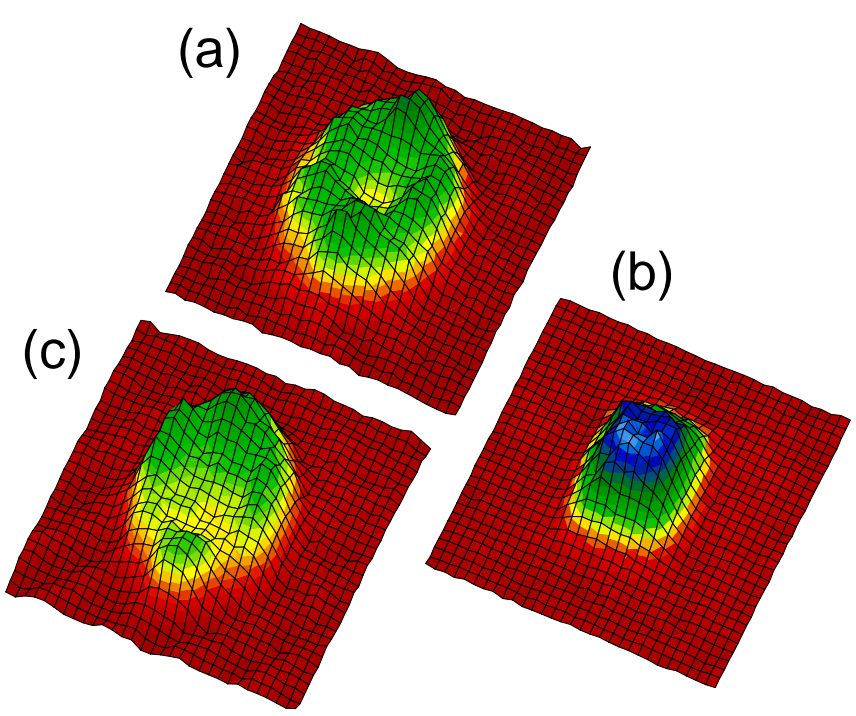

FIG. 2. (Color) (a) The image of the $|1\rangle$ condensate exhibits a "crater," corresponding to a shell in which the $|2\rangle$ atoms (b) reside. For this trap, $\nu_{z}=47 \mathrm{~Hz}$ with zero relative sag. By changing the strength of the magnetic quadrupole field we can introduce a nonzero relative sag, which shifts the location of the "crater" (c). (Each square in this post-expansion image is $136 \mu \mathrm{m}$ on a side.)

By adjusting these parameters, we can change the sign of the relative sag or cause it to vanish [19] while preserving (to first order) the same radial $\left(\nu_{r}\right)$ and axial $\left(\nu_{z}=\sqrt{8} \nu_{r}\right)$ trap oscillation frequencies.

In a first experiment, we choose a trap that has zero relative sag $\left(\nu_{z}=47 \mathrm{~Hz}\right)$ and transfer $50 \%$ of the atoms to the $|2\rangle$ state with a $\sim 400 \mu$ s pulse. When $T=30 \mathrm{~ms}$, we observe a "crater" in the image of the $|1\rangle$ atoms (Fig. 2a). The "crater" corresponds to a region occupied by the $|2\rangle$ atoms (Fig. 2b), indicating that the $|1\rangle$ atoms have formed a shell about the $|2\rangle$ atoms. This is consistent with the theoretical observation that it is energetically favorable for the atoms with the larger scattering length $(|1\rangle)$ to form a lower-density shell about the atoms with the smaller scattering length $(|2\rangle)[9]$. At longer times the condensates separate from one another radially [20].

In order to explore the boundary between the two condensates, we perform a series of experiments in a trap in which we displace the trapping potentials such that the minimum of $V_{2}$ is $0.4 \mu \mathrm{m}$ lower than that of $V_{1}$, or approximately $3 \%$ of the (total) extent of the combined density distribution in the vertical direction. The subsequent time-evolution of the system is shown in Figs. 3 and 4. The two states almost completely separate (Fig. 3a-c) after $10 \mathrm{~ms}$; they then "bounce" back until at $T=25 \mathrm{~ms}$ the centers-of-mass are once more almost exactly superimposed (Fig. 1), although a distinctive (and reproducible) vertical structure has formed (Fig. Bdd-e-f). By $T=65 \mathrm{~ms}$, the system has apparently reached a steady 
1)
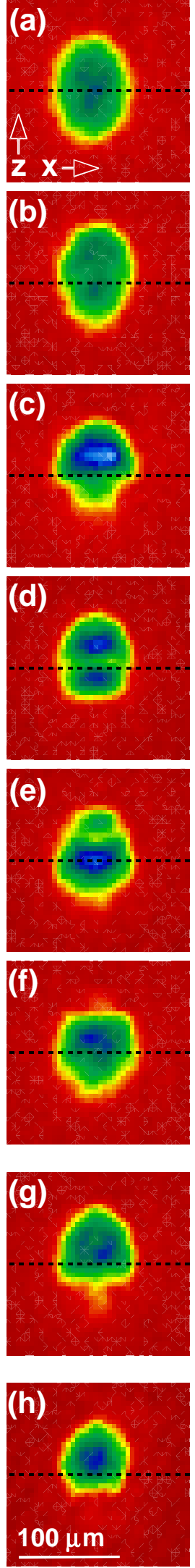

$|2\rangle$
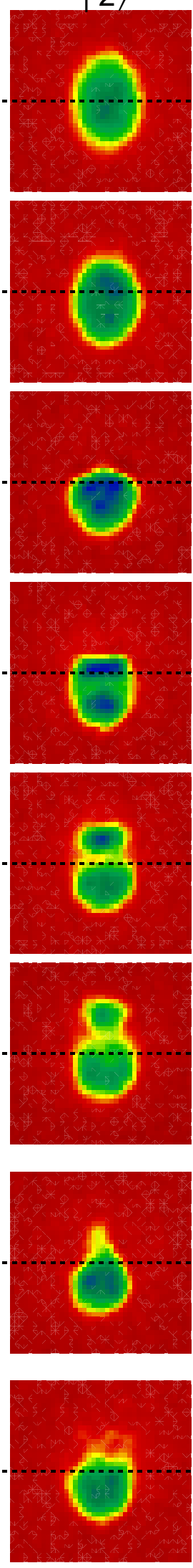
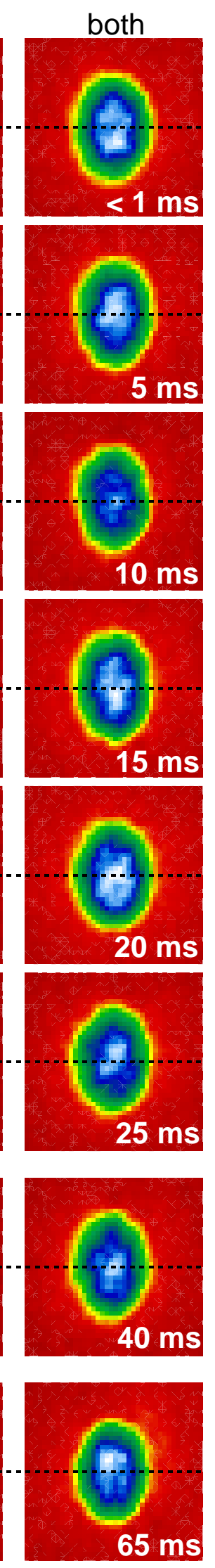

FIG. 3. (Color) Time-evolution of the double-condensate system with a relative sag of $0.4 \mu \mathrm{m}$ (3\% of the width of the combined distribution prior to expansion) and a trap frequency $\nu_{z}=59 \mathrm{~Hz}$.

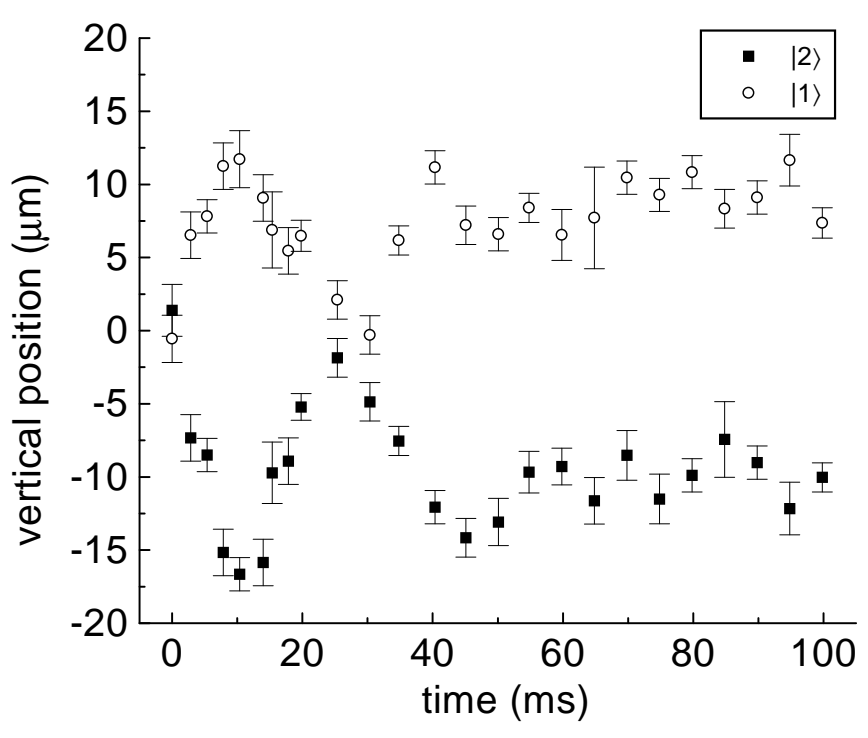

FIG. 4. The relative motion of the centers-of-mass of the two condensates under the same conditions as those in Fig. 3.

state (Fig. 3g-h, Fig. (4) in which the separation of the centers-of-mass is $20 \%$ of the extent of the cloud. From these images we observe: (i) the fractional steady-state separation of the expanded image is large compared to the fractional amount of applied symmetry breaking, as we expect for a repulsive interspecies potential; (ii) the placid total density profile (rightmost column of Fig. 3) betrays little hint of the underlying violent rearrangement of the component species; and (iii) the component separation is highly damped, although it is not yet certain what mechanism [21] is responsible. With respect to the damping, the excitation is in no sense small and may therefore be poorly modeled by theories that treat the low-lying, small-amplitude excitations [10] of double condensates.

Finally, we show the optical density as a function of relative number and position on the condensate vertical axis in order to better appreciate the amount of overlap between the two states at $T=65 \mathrm{~ms}$ (Fig. 回), which remains substantial despite the underlying separation. Each plot is averaged across a $\sim 14 \mu \mathrm{m}$ wide vertical cut through the centers of the two condensates. From the overlap shown, one could determine the magnitude of the interspecies scattering length $a_{12}$ by comparison to numerical solutions of the Gross-Pitaevskii equations (1) for our trapping conditions. Such a calculation is beyond the scope of the present work.

In related work, we have read out the relative quantum phase of the condensates in the overlap region with a sec- 

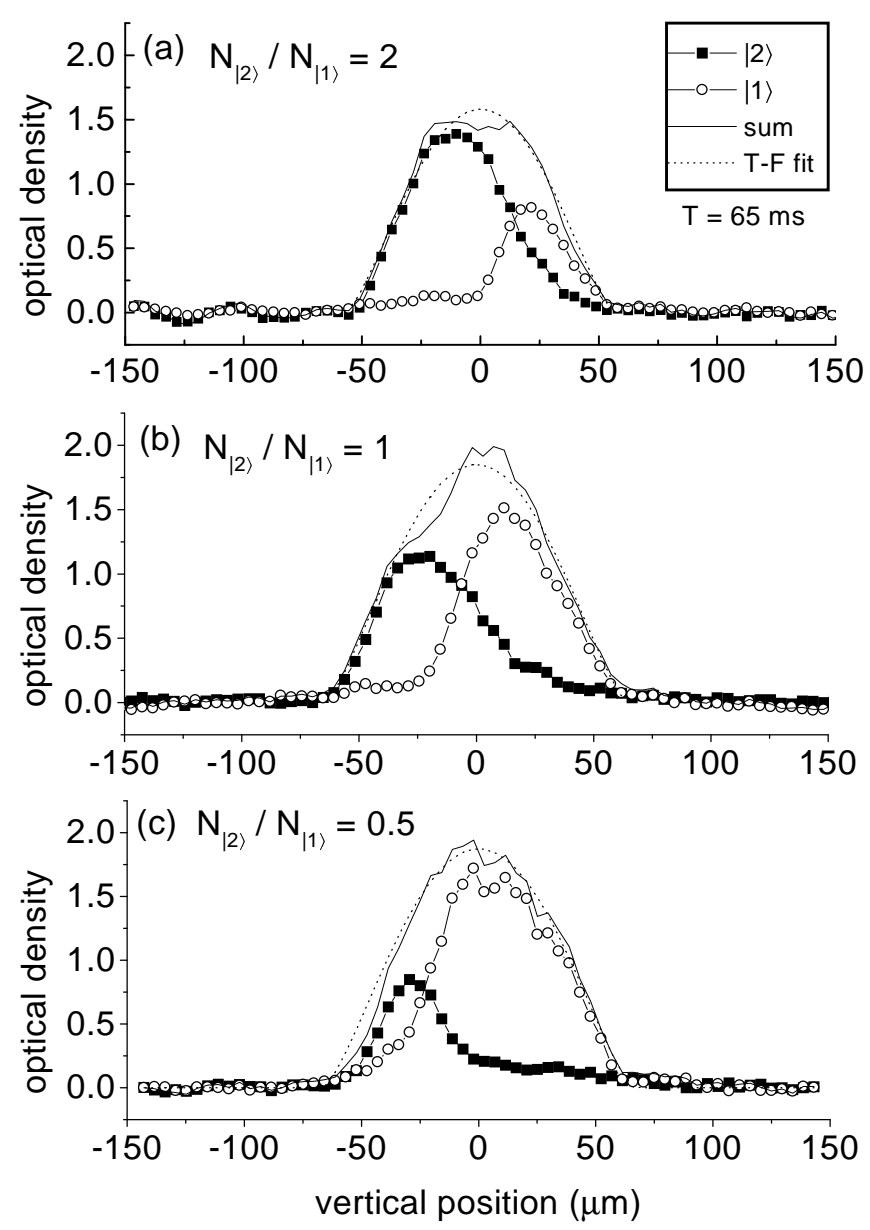

FIG. 5. Vertical cross-sections of the density profiles at $T=65 \mathrm{~ms}$ for different relative numbers of atoms in the two states. The combined density distribution (solid line) is shown for comparison to the Thomas-Fermi parabolic fit (dashed line). The trap parameters are the same as those in Fig. 3 .

ond two-photon pulse, making use of Ramsey's method of separated oscillatory fields. We expect the presence of a weak cw two-photon drive, resonant in the overlap region, to result in phase-locking and phase-sensitive currents analogous to the Josephson effect in superconductors 22]. We will discuss the phase-evolution of the condensates in a future publication.

We gratefully acknowledge useful conversations with the other members of the JILA BEC collaboration, in particular those with Chris Greene and John Bohn. This work is supported by the ONR, NSF, and NIST.

* Quantum Physics Division, National Institute of Stan- dards and Technology.

[1] M. H. Anderson et al., Science 269, 198 (1995).

[2] K. B. Davis et al., Phys. Rev. Lett. 75, 3969 (1995).

[3] C. C. Bradley, C. A. Sackett, and R. G. Hulet, Phys. Rev. Lett. 78, 985 (1997).

[4] C. J. Myatt et al., Phys. Rev. Lett. 78, 586 (1997).

[5] D. M. Stamper-Kurn et al., Phys. Rev. Lett. 80, 2027 (1998).

[6] I. M. Khalatnikov, Zh. Eksp. Teor. Fiz. 32, 653 (1957) [Sov. Phys. JETP 5, 542 (1957)]; I. M. Khalatnikov, Pis'ma Zh. Eksp. Teor. Fiz. 17, 534 (1973) [JETP Lett. 17, 386 (1973)]; Yu. A. Nepomnyashchǐ̌, Zh. Eksp. Teor. Fiz. 70, 1070 (1976) [Sov. Phys. JETP 43, 559 (1976)].

[7] E. D. Siggia and A. E. Ruckenstein, Phys. Rev. Lett. 44, 1423 (1980).

[8] T.-L. Ho and V. B. Shenoy, Phys. Rev. Lett. 77, 3276 (1996); B. D. Esry, C. H. Greene, J. P. Burke, Jr., and J. L. Bohn, Phys. Rev. Lett. 78, 3594 (1997); C. K. Law, H. Pu, N. P. Bigelow, and J. H. Eberly, Phys. Rev. Lett. 79, 3105 (1997).

[9] H. Pu and N. P. Bigelow, Phys. Rev. Lett. 80, 1130 (1998).

[10] E. V. Goldstein and P. Meystre, Phys. Rev. A 55, 2935 (1997); T. Busch, J. I. Cirac, V. M. Pérez-García, and P. Zoller, Phys. Rev. A 56, 2978 (1997); R. Graham and D. Walls, Phys. Rev. A 57, 484 (1998); B. D. Esry and C. H. Greene, Phys. Rev. A 57, 1265 (1998); H. Pu and N. P. Bigelow, Phys. Rev. Lett. 80, 1134 (1998).

[11] M. R. Matthews et al., e-print cond-mat/9803310.

[12] W. Petrich, M. H. Anderson, J. R. Ensher, and E. A. Cornell, Phys. Rev. Lett. 74, 3352 (1995).

[13] M. Holland and J. Cooper, Phys. Rev. A 53, R1954 (1996).

[14] J. P. Burke, Jr. (private communication).

[15] E. Zaremba, A. Griffin, and T. Nikuni, e-print condmat/9705134.

[16] B. D. Esry (private communication).

[17] J. L. Bohn (private communication).

[18] D. S. Hall et al., Proc. SPIE 3270 (in press).

[19] The relative sag can also be adjusted in a static magnetic trap by making use of the Breit-Rabi dependence of the magnetic moments on the applied bias field.

[20] We believe this effect to be related to asymmetries in the trapping fields.

[21] P. A. Ruprecht, M. J. Holland, K. Burnett, and M. Edwards, Phys. Rev. A 51, 4704 (1995); L. P. Pitaevskii and S. Stringari, Phys. Lett. A 235, 398 (1997); Yu. Kagan, E. L. Surkov, and G. V. Shlyapnikov, Phys. Rev. Lett. 79, 2604 (1997); L. P. Pitaevskii, Phys. Lett. A 229, 406 (1997); W. V. Liu, Phys. Rev. Lett. 79, 4056 (1997); P. O. Fedichev, G. V. Shlyapnikov, and J. T. M. Walraven, Phys. Rev. Lett. 80, 2269 (1998); S. Giorgini, Phys. Rev. A 57, 2949 (1998); T. Nikuni and A. Griffin, e-print cond-mat/9711036.

[22] B. D. Josephson, Phys. Lett. 1, 251 (1962); J. Javanainen, Phys. Rev. Lett. 57, 3164 (1986); I. Zapata, F. Sols, and A. J. Leggett, Phys. Rev. A 57, R28 (1998); J. Williams and M. Holland (private communication). 